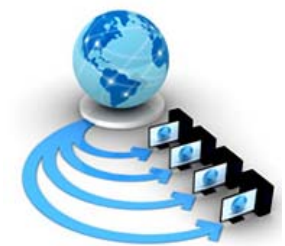

Volume 8, No. 9, November-December 2017

International Journal of Advanced Research in Computer Science

RESEARCH PAPER

Available Online at www.ijarcs.info

\title{
ADVANCE AUTOMATED RAILWAY CROSSING SYSTEM
}

\author{
Akansh Saxena \\ UG,Electronics and Communication Engineering \\ Birla Institute Of Applied Sciences \\ Bhimtal, Nainital, India
}

\author{
Pawan Singh \\ UG,Electronics and Communication Engineering \\ Birla Institute Of Applied Sciences \\ Bhimtal, Nainital, India
}

\begin{abstract}
This paper presents a railway crossing system which is an advanced version of traditional automated railway crossing system. This system will not only help to improve railway crossing system working but it can also control the latter situations of traffic. It can easily replace the traditional ways involved and is less prone to errors. This paper presents the complete working involved in the process, requirements and the problems that are faced. It includes hardware components like Arduino UNO, servo motor, photoresistor, 7-segments display, push buttons, GPRS/GSM module and for sending data we hosted a website on Amazon Web Services(AWS). This paper presents a system that can be easily installed all over the country with low establishment cost and good efficiency.
\end{abstract}

Keyword: Arduino, Railway Crossing, Servo Motor, Photoresistor, Amazon Web Services(AWS), Internet of Things(IoT)

\section{INTRODUCTION}

This paper proposes a system that is an advanced version of the automatic railway crossing system used. There are basically two kinds of system that are currently used for the railway crossing, one is the most common way in which orders are sent from the nearest railway station and controller has to shut down the barriers by himself, this includes human work whereas the second way is a technical approach in which sensors are installed near the crossing and if they sense any train coming then the system will automatically shut down the barriers. The current status of the system and values from the sensor will be sent to the administrator through a website which can be hosted on Amazon Web Services(AWS). The administrator will be someone working on the nearest railway station, he can be a controller from nearest station and can handle the system in case of emergency or if anything goes wrong. Our approach is an advanced version of the technical approach. The traditional technical approach includes synchronous working between railway tracks and a road. The traditional system does its work efficiently but the problem arises on a normal routine after the train passes, as it passes barriers get open and vehicles are allowed to pass but there's no arrangement done for pedestrians. No proper arrangement at level crossings is the biggest reason for the accidents, accounting for $40 \%$ of train accidents and $66 \%$ of fatalities, telling the story of the railway's failure to man these crossings or build road-over-bridges and road-under-bridges. There are 30,348 level crossings in India, of which around $40 \%(11,563)$ are unmanned [1]. This system is made to solve that problem. This system includes synchronous working between railways track, road, and a pedestrian path. It provides enough time to vehicles and pedestrians so that they can cross the road and the conditions can be changed according to need.

\section{Advantage OF System}

The two foremost advantage of the system is that it works for more than two paths i.e. it also includes pedestrian path and it needs no surveillance which means this system can be easily installed in $40 \%$ of the railway crossings in India which are unmanned. Basically, it is always a problem that pedestrians don't get enough space and time to cross the track so they have to hop on the road which is not safe for them and for others too. This also leads to traffic jams and sometimes some serious accidents that lead to a big problem on a busy road and sometimes cost human lives. One solution of this is making overhead bridges for pedestrians but this solution is not economical and feasible in remote areas. This system provides an alternative solution to these problems. It is an efficient, economical and better than previous systems in many ways. The system can also be controlled by the administrator from nearest station so it provides both ways of controlling. The system is connected to the internet so it an Internet of Things(IoT) system. Fig-1 shows how everything interacts with the system. 


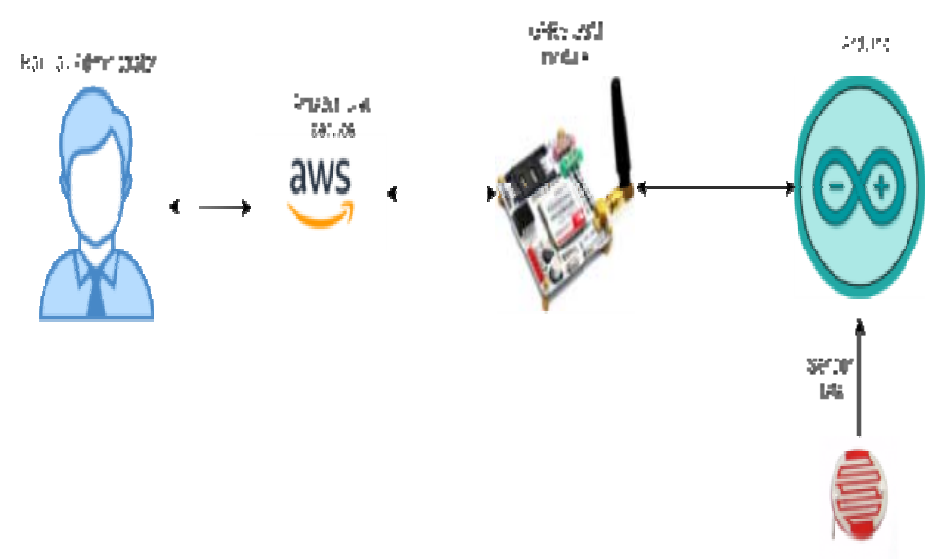

Fig-1 System Architecture

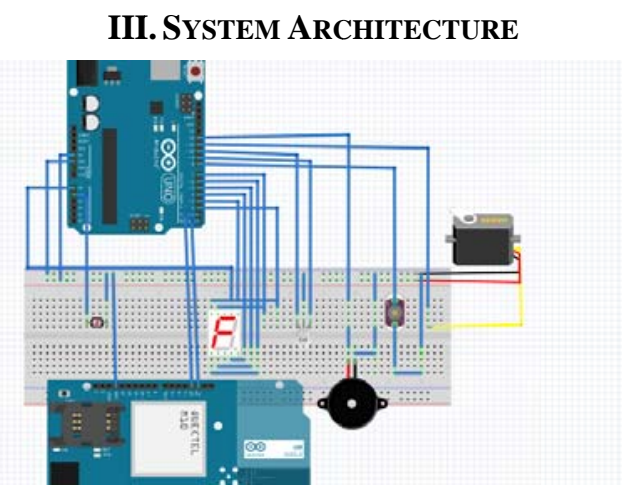

Fig-2 Pin Diagram[2]

Fig-2 shows the pin diagram of the system. The components used for the miniature system are Arduino UNO [3], GPRS/GSM module, servo motor, RGB LED, buzzer[4], breadboard, photoresistor[5], push button, 7-segment display [6] and wires for connection.

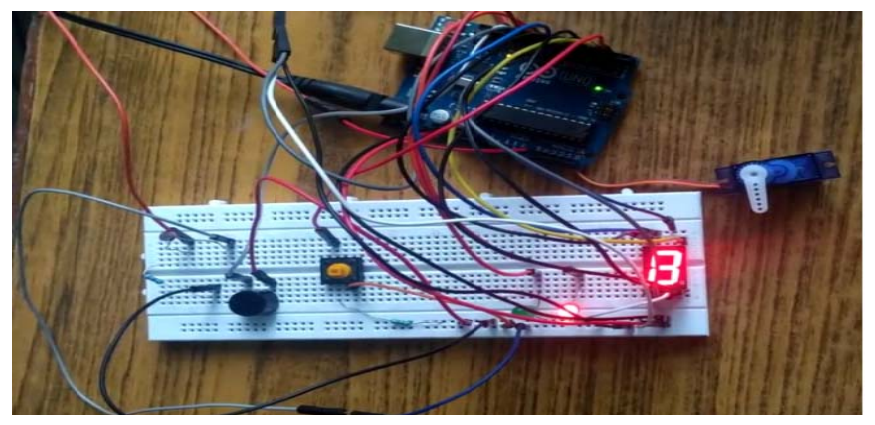

Fig-3 Practical Setup

Arduino UNO acts as the controller that controls all the working of the system. It has the program booted in it and runs with least error. RGB LED is used to indicate the status of pedestrian path i.e. red color indicates that the path is closed and green color shows that the path is open and pedestrians are allowed to cross the road. 7-segment display works as the (c) 2015-19, IJARCS All Rights Reserved counter that counts the time from 0 seconds to 9 seconds, the servo motor is a barrier that indicates the status of the road, if the barrier is vertical then the road is open and if the barrier is horizontal then the road is closed. A photoresistor is one of the most important parts of this system which acts as a sensor that checks the arrival of the train. The push button is used as a button that will be installed on the pedestrian path. GPRS/GSM module[7] provides internet connection to the system so that data can be sent to the hosted website and system can be controlled accordingly.

\section{FLOWCHART}

The flowchart in Fig-4 shows the working of a system in pictorial form. Initially, the road is open and barriers are open this implies that the pedestrian path is closed indicated by glowing red light. If sensors sense the arrival of the train then roads get closed, barriers go down and the pedestrian path remains closed. After the train leaves, there are two cases, the default case is road opens and barriers open and pedestrian remain close where the other

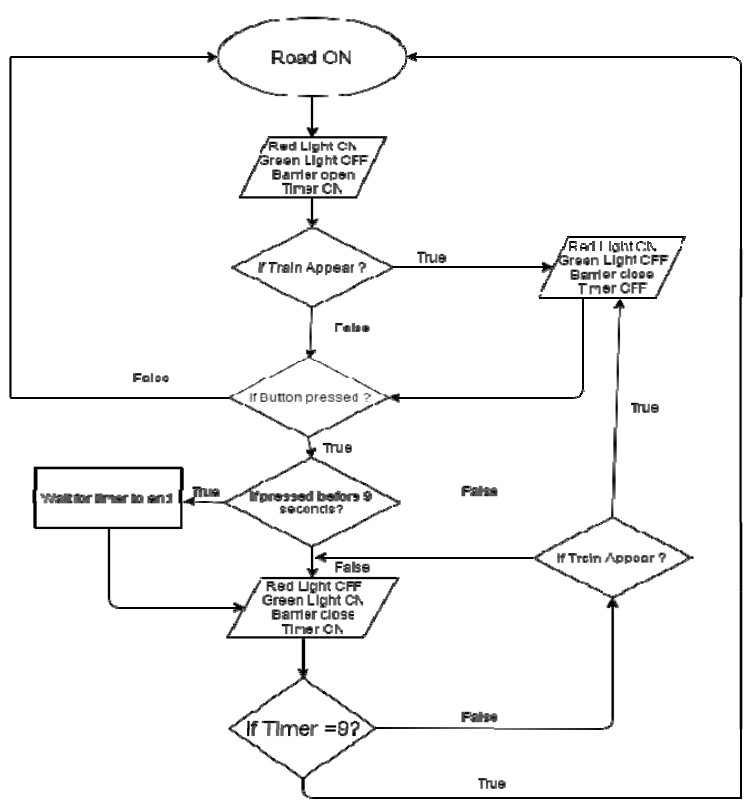

Fig.-4 Flow Chart

the case is if during the passage of train someone presses the button then after the train passes pedestrian path is open indicated by glowing green light and in this case vehicles are not allowed to pass for the time pedestrian path is open. Every time when the pedestrian path is open, they get 9 seconds to cross the road and after this pedestrian path is closed and the road is open allowing vehicles to pass. During the road is open the pedestrian path doesn't open until required which means that pedestrians have to decide when they want the pedestrian path to open. This happens so, when road gets open then a counter starts counting from 0 to 9 and if someone presses the button before 9 seconds then nothing happens until 9 seconds are completed and as soon as 9 seconds gets over, road gets 
closed and pedestrians are allowed to pass but if the button is pressed sometime after 9 seconds then road gets closed immediately and pedestrian paths open for 9 seconds. But during all this process, sensors sense any train coming then both road and pedestrian path is closed allowing passage of the train.

\section{Working Principle}

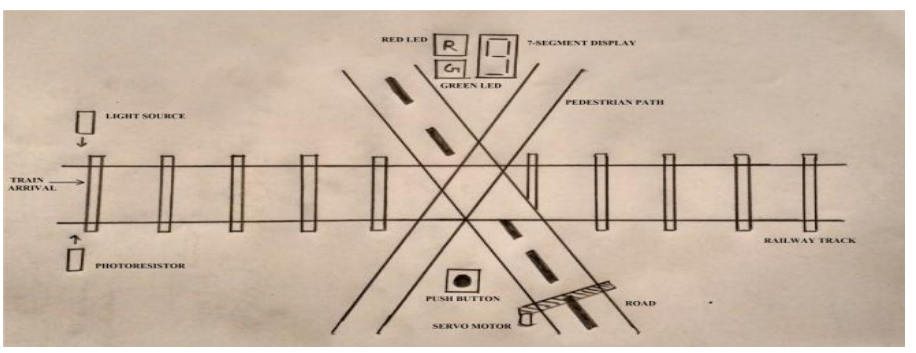

Fig-5 Arrangement

Fig-5 shows the arrangement of the system. Photoresistor will be installed around 1-2 km away from crossing the reason being is, it will close the barriers prior to the arrival of a train. It can be risk-taking in installing the sensor close to the crossing. There will be a light source opposite to the photoresistor, the light source can be a LASER[8] which will always focus some light on the photoresistor and when the train will arrive this connection will be broken. Photoresistor works on a simple phenomenon, it produces some resistance when light falls on it and this value of resistance is inversely proportional to the intensity of light i.e. resistance decreases when intensity increases. When there's no hindrance in light falling on photoresistor then it gives some reading to microcontroller but when a train comes in between the reading value drops and microcontroller takes action and closes the barrier and glowing red light, turning off the timer and buzzer will start beeping as the sign of warning. When the train passes, the microcontroller will check for the button input, if it is pressed during train was passing then it opens pedestrian path keeping road close but if the button is not pressed then the road opens and vehicles will we allowed to pass keeping pedestrian path close. Then during this the condition when road is open, microcontroller will again check for button input and if it is pressed before 9 seconds then microcontroller waits for completion of minimum time of 9 seconds and then closes the road, opening the pedestrian path but if the button is pressed after 9 seconds then microcontroller immediately opens pedestrian path, closing the road. While the pedestrian path is open, the buzzer will beep continuously for 9 seconds until a pedestrian path is closed.

All data is sent to the administrator with the help of the website, he can look after the status of the system and if it is working correctly or not and if there's any change to make to working then he can control the system from the railway station only. In case of emergency, like if the train is coming and sensor didn't read that, then the administrator can shut down the road and pedestrian path by just clicking one dedicated button to the website.

\section{Result AND ANAlysis}

This project gives us an idea of how a combination of simple microcontroller, sensors, and other components can be used to build a railway track crossing system. The system works efficiently on the miniature level. It has lots of advantages in comparison to traditional methods. Being an Internet of Things(IoT) device, the system is connected to the internet so it has fewer chances of error, in case of any fault then it can be easily controlled by the administrator.

\section{CONCLUSIONS}

In this paper, we have introduced a system which is efficient and cost-effective which can be implemented easily. It can help in reducing human error and manpower in comparison to traditional systems. It can also save human lives. For the advancement of the system it is also connected to GPRS/GSM module which will provide internet access and we can host a website on AWS[9] where all data will be sent to an administrator that can access the website from nearest railway station such that, if something goes wrong or in case of emergency he can shut down everything just by clicking one button on the website.

\section{ACKNOWLEDGEMENT}

We would like to express our special thanks of gratitude to our colleagues Mitul Tyagi, Ishant Garg, Himanshu Joshi for giving required support in the project. We have taken efforts in this project. However, it would not have been possible without the kind support of Dr. Sandesh Tripathi [10], H.O.D Department of Computer Science and Engineering, Birla Institute of Applied Sciences, Nainital. Therefore, we would like to extend our gratitude for his guidance and constant supervision throughout the endeavor and also for the comments that greatly improved this manuscript

\section{REFERENCES}

[1] Train_accident:https://timesofindia.indiatimes.com/india/6 6-of-accident-fatalities-at-levelcrossings/articleshow/38984972.cms

[2] Fritzing:http://fritzing.org/home/

[3] Arduino UNO: https://www.arduino.cc

[4] Buzzer_Arduino:https://www.arduino.cc/reference/en/lan guage/functions/advanced-io/tone/

[5] Photoresistor:https://playground.arduino.cc/Learning/Phot oResistor

[6] 7_segement_dispaly:https://circuitdigest.com/microcontro ller-projects/7-segment-display-interfacing-with-arduino

[7] https://electronicsforu.com/resources/gsm-module

[8] https://en.wikipedia.org/wiki/Laser

[9] https://aws.amazon.com/

[10]_sandeshtripathi@birlainstitute.co.in 